\title{
FNCLCC Sarcoma Grade 3
}

National Cancer Institute

\section{Source}

National Cancer Institute. FNCLCC Sarcoma Grade 3. NCI Thesaurus. Code C9029.

A sarcoma with a total score of 6 or higher according to the FNCLCC guidelines. 